\title{
Video Article \\ Effect of Artificial Tear Formulations on the Metabolic Activity of Human Corneal Epithelial Cells after Exposure to Desiccation
}

\author{
Rekha Rangarajan ${ }^{1}$, Howard A. Ketelson ${ }^{1}$, Richard Do ${ }^{2}$, David J. McCanna ${ }^{2}$, Adeline Suko ${ }^{2}$, Daryl Enstone ${ }^{2}$, Lakshman N. Subbaraman ${ }^{1}$, \\ Jaya Dantam², Lyndon W. Jones ${ }^{2}$ \\ ${ }^{1}$ Alcon Vision, LLC \\ ${ }^{2}$ Centre for Ocular Research and Education (CORE), School of Optometry \& Vision Science, University of Waterloo
}

Correspondence to: David J. McCanna at djmccann@uwaterloo.ca

URL: https://www.jove.com/video/60812

DOI: doi:10.3791/60812

Keywords: Medicine, Issue 159, metabolic activity, dry eye, artificial tear formulation, desiccation, human corneal epithelial cells, metabolic dye

Date Published: 5/2/2020

Citation: Rangarajan, R., Ketelson, H.A., Do, R., McCanna, D.J., Suko, A., Enstone, D., Subbaraman, L.N., Dantam, J., Jones, L.W. Effect of Artificial Tear Formulations on the Metabolic Activity of Human Corneal Epithelial Cells after Exposure to Desiccation. J. Vis. Exp. (159), e60812, doi:10.3791/60812 (2020).

\section{Abstract}

Artificial lipid-containing tear formulations are developed to reduce tear evaporation by the restoration of a deficient tear lipid layer. Artificial tear formulations that prevent cell desiccation will result in ocular surface protection and the maintenance of cell metabolic activity. During dehydration, cells undergo the process of loss of metabolic activity and subsequently cell death. This work describes a method for assessing the efficacy of artificial tear formulations. The metabolic dye (i.e., alamarBlue) changes from a low fluorescent molecule resazurin to a fluorescent molecule resorufin in viable cells. The biological performance of an artificial tear formulation is measured as the ability of the formulation to (a) maintain cell viability and (b) provide cell protection from desiccation. Growth media and saline are used as controls for the cell viability/ desiccation tests. Cells are incubated with test solutions for $30 \mathrm{~min}$ and then desiccated for 0 or 5 min at $37{ }^{\circ} \mathrm{C}$ and $45 \%$ relative humidity. Cell metabolic activity after initial exposure and after cell desiccation is then determined. The results show the comparative effects of eye drop formulations on cell metabolic activity and desiccation protection. This method can be used to test dry eye formulations that are designed to treat individuals with evaporative dry eye.

\section{Introduction}

Many different ingredients in multiple-dose ophthalmic solutions are needed to maintain stability, $\mathrm{pH}$, osmolarity, and efficacy. However, some chemicals such as preservatives or surfactants in ocular solutions can cause damage to the corneal epithelium ${ }^{1,2}$. Cell viability tests using human corneal epithelial cells (HCEC) can be performed to assess the potential damage caused by these various ingredients in ophthalmic formulations. One of the in vitro assays that is particularly useful for assessing cell damage is the alamarBlue assay ${ }^{3}$. In this assay, the metabolic dye (i.e., alamarBlue) contains resazurin, which functions as a cell viability indicator. During cellular respiration, resazurin is reduced to resorufin by accepting electrons ${ }^{4}$. The reduction of resorufin causes a change from a non-fluorescent molecule to a fluorescent molecule that can be detected using a spectrofluorometer ${ }^{5}$.

This investigation uses two different treatments of the corneal epithelial cells to determine how dry eye products may perform in an in vitro model. In the first evaluation, the cells are treated with these products for $30 \mathrm{~min}$. After treatment, the cells are then evaluated for metabolic activity immediately after exposure to the dry eye products and after a recovery time interval. This assessment determines the effect of these products on cells prior to desiccation in a humidity chamber. Solution preservatives, surfactants or buffers in these solutions may have some cytotoxic effects on the cells that can be detected using the metabolic dye.

The second evaluation determines the metabolic activity of the cells after exposure to the dry eye products and desiccation. In this assessment, if the product treatment offers protection to the cells from desiccation damage, the metabolic activity of the cells will be maintained. Cell protection from the harmful effects of desiccation may occur if the dry eye product can coat the cells with lipids or the product may absorb liquid from the surroundings adding moisture to the cells.

This protocol is designed to simulate severe conditions of environmental stress on cells. Other studies have used various models to create this external stress. Some studies have dried the cells in laminar flow hoods ${ }^{6,7,8}$, whereas others have used room temperature and various relative humidity $(\mathrm{RH})$ values ${ }^{9,10,11}$. A method of simulating dry eye conditions is to increase the osmolarity of the incubating solution ${ }^{12,13}$. Another in vitro method for simulating adverse environmental conditions is to add cytokines to the cell media to simulate the tear fluid of dry eye patients ${ }^{14}$. Although these tests are interesting models that can evaluate how chemicals reduce osmotic stress or stimulate the immune system, these models do not directly show how chemical formulations can protect cells from desiccation stress.

The desiccation model uses a temperature/humidity chamber $37{ }^{\circ} \mathrm{C}$ and $45 \% \mathrm{RH}$. It simulates environmental conditions that can cause evaporation from the ocular surface. Simulations of other extreme environmental conditions such as the low humidity found in airplane cabins ${ }^{15}$ or in indoor environments during the winter months ${ }^{16}$ can also be performed using this method. 
Cell culture modeling is used to simulate the effect of desiccation stress on the human cornea. In order to detect cell stress, cell viability testing is undertaken to determine the impact on cell health. Several different physiological tests can be performed on cells to determine if they are stressed $^{17}$. The analysis performed in this test procedure uses the commercially available metabolic dye (Table of Materials). It has the advantage over many other cell stress tests in that it is non-toxic, soluble in growth media and can evaluate cells without cell fixation ${ }^{18}$. In this procedure the cells are tested for metabolic activity immediately after each treatment and a second set of cells are placed back into growth media and evaluated after $18 \mathrm{~h}$ recovery. The reason for testing immediately and then after a recovery period is to identify cell damage that causes immediate effects on metabolic activity and cell damage that causes delayed effects. Also, it provides an opportunity to evaluate the impact of these formulations on short-term versus long-term damage and the ability of the formulations to recover/not recover from the initial insult.

This investigation is used to compare various lipid-containing dry eye products for their effect on HCEC metabolic activity with and without desiccation. The ingredients in the dry eye products tested are described in Table of Materials. The ingredients in solution \#1 are carboxymethylcellulose sodium ( $0.5 \%)$, glycerin (1\%), and polysorbate $80(0.5 \%)$, in solution \#2 they are light mineral oil $(1.0 \%)$ and mineral oil $(4.5 \%)$, and in solution \#3 the lipid is mineral oil and propylene glycol is the demulcent. These lipids are the formulation components that are expected to provide protection of the HCEC from desiccation.

\section{Protocol}

\section{Human corneal epithelial cell culture}

1. Grow immortalized $\mathrm{HCEC}^{19}$ in collagen-coated $75 \mathrm{~cm}^{2}$ flasks with $20 \mathrm{~mL}$ of Dulbecco's modified Eagle medium/nutrient mixture F-12 (DMEM/ F12) containing $10 \%$ fetal bovine serum (FBS) and $1 \%$ penicillin/streptomycin at $37^{\circ} \mathrm{C}$ with $5 \% \mathrm{CO}_{2}$. NOTE: Shaking is not required.

2. Change the media every $2-3$ days.

\section{Preparation of cells for testing}

1. After the cells are almost confluent remove culture media

2. Add 4-6 $\mathrm{mL}$ of cell disassociation solution to each $75 \mathrm{~cm}^{2}$ flasks. Incubate the cells at $37^{\circ} \mathrm{C}$ until the cells detach (approximately $20-30$ min), checking under the microscope periodically.

3. Add 2-6 $\mathrm{mL}$ of DMEM/F12 containing $10 \%$ FBS to each $75 \mathrm{~cm}^{2}$ flask.

4. Transfer the contents of the flask to a $50 \mathrm{~mL}$ centrifuge tube.

5. Centrifuge the cells at $450-500 \times g$ for $5 \mathrm{~min}$. Pipet out the supernatant and resuspend the cells in pre-warmed media.

6. Count the cells using a hemocytometer. Calculate the volume of media that contains a total of $1 \times 10^{5}$ cells

7. Add $1 \times 10^{5}$ cells to each well of a 48 well collagen-1 coated culture plate. Add enough media to the well so that the final volume of media in the well is $0.5 \mathrm{~mL}$ of culture DMEM/F12 with $10 \%$ FBS.

NOTE: An artificial tear formulation may cause cytotoxicity that can be measured by a decrease in the metabolic activity of HCEC. The consistency of the data is dependent on adding the same number of HCEC to each well when seeding. The culture concentration that is recommended for 48 well plates is $1 \times 10^{5}$. Because mammalian cells can settle in the centrifuge tube after resuspending, it is recommended to resuspend the cells by agitation frequently while seeding the plates with cells so that the cell suspension has the HCEC equally distributed.

8. Incubate the cells at $37^{\circ} \mathrm{C}$ and $5 \% \mathrm{CO}_{2}$ for $24 \mathrm{~h}$.

\section{No desiccation protocol}

1. Control procedure

1. After the $24 \mathrm{~h}$ incubation remove the culture media from the plate.

2. Immediately treat with $150 \mu \mathrm{L}$ of a test formulation and media control solution.

3. Incubate the cells at $37{ }^{\circ} \mathrm{C}$ and $5 \% \mathrm{CO}_{2}$ for $30 \mathrm{~min}$.

4. Remove the test solutions from the cells.

5. Add $0.5 \mathrm{~mL}$ of $10 \%$ metabolic dye solution to test for metabolic activity. Incubate the cells for another $4 \mathrm{~h}$ at $37^{\circ} \mathrm{C}$ and $5 \% \mathrm{CO}_{2}$.

6. After the $4 \mathrm{~h}$ incubation period, remove $100 \mu \mathrm{L}$ of metabolic dye solution from each well and place each $100 \mu \mathrm{L}$ into a well of a 96 well plate.

7. Measure the fluorescence of each well using a fluorescent plate reader (Table of Materials). Set the excitation wavelength at $540 \mathrm{~nm}$ and the emission wavelength at $590 \mathrm{~nm}$.

2. No desiccation protocol (recovery procedure)

1. Repeat steps 3.1.1-3.1.4.

2. Add $0.5 \mathrm{~mL}$ of DMEM/F12 media to each well.

3. Incubate one set of cultures for $18 \mathrm{~h}$ at $37^{\circ} \mathrm{C}$ and $5 \% \mathrm{CO}_{2}$.

4. Remove the media and add $0.5 \mathrm{~mL}$ of $10 \%$ metabolic dye solution. Repeat steps 3.1 .6 and 3.1.7.

\section{Desiccation protocol}

1. Control procedure

1. After the $24 \mathrm{~h}$ incubation (step 2.8), remove the culture media from the plate.

2. Immediately treat with $150 \mu \mathrm{L}$ of a test formulation and media control solution. 
3. Incubate the cells at $37^{\circ} \mathrm{C}$ and $5 \% \mathrm{CO}_{2}$ for $30 \mathrm{~min}$.

4. Remove the test solutions from the cells.

5. Place the cells in a $37^{\circ} \mathrm{C}$ and $45 \%$ RH chamber for 5 min to desiccate the cells.

6. Add $0.5 \mathrm{~mL}$ of $10 \%$ metabolic dye solution to test metabolic activity. Incubate the cells for another $4 \mathrm{~h}$ at $37{ }^{\circ} \mathrm{C}$ and $5 \% \mathrm{CO}_{2}$.

7. After the $4 \mathrm{~h}$ incubation period, remove $100 \mu \mathrm{L}$ of metabolic dye solution from each well and place each $100 \mu \mathrm{L}$ into a well of a 96 well plate.

8. Measure the fluorescence of each well using a fluorescent plate reader. Set the excitation wavelength at $540 \mathrm{~nm}$ and the emission wavelength at $590 \mathrm{~nm}$.

2. Recovery procedure

1. Repeat steps 4.1.1-4.1.5.

2. Add $0.5 \mathrm{~mL}$ of DMEM/F12 media to each well.

3. Incubate one set of cultures for $18 \mathrm{~h}$ at $37^{\circ} \mathrm{C}$ and $5 \% \mathrm{CO}_{2}$.

4. Remove the media and add $0.5 \mathrm{~mL}$ of $10 \%$ metabolic dye solution. Repeat steps 4.1 .7 and 4.1.8.

\section{Data analysis}

1. Calculate the average fluorescence of the media control. Calculate the percent viability of the sample using the follow formula:

Percent viability of the sample $=$ test sample fluorescence/media control fluorescence $\times 100$

NOTE: The media control average is $100 \%$ viability. The metabolic dye by itself has a small amount of fluorescence associated with it. The fluorescence of the metabolic dye reagent can be subtracted from the control and test sample fluorescence prior to calculating \% viability of the test samples to the media control.

2. Perform a normality test and a test for equal variances on the control and test samples.

3. If the normality test passes and the variances are equal then perform a one-way ANOVA. To identify significant differences between specific groups perform a pairwise comparisons post hoc test (i.e., Tukey or Dunnett's multiple comparisons tests).

4. If the normality test passes and the variances are different then perform the Welch's ANOVA. To identify significant differences between specific groups perform a pairwise comparisons post hoc test (i.e., Dunnett's or Games-Howell multiple comparisons tests).

5. If the normality test fails use a nonparametric test (i.e., Kruskal-Wallis test). To identify significant differences between specific groups perform a pairwise comparisons post hoc test (i.e., Dunn's test).

\section{Representative Results}

Results from a study comparing three dry eye formulations are shown in Figure 1. This figure shows that there are differences in the effect the three products, solution \#1, solution \#2 and solution \#3 (Table of Materials) have on the viability of HCEC. Solution \#1 and solution \#2 had a significant effect on the metabolic activity of HCEC before desiccation. This means that there are formulation components in these products that can disrupt the metabolic activity of the HCEC. The additional drop in cell metabolic activity that occurred after $18 \mathrm{~h}$ recovery of cells exposed to solution \#1 shows that HCEC were injured initially and after $18 \mathrm{~h}$ the injury was not repaired. In comparison, solution \#3 had only a mild effect on the metabolic activity of the HCEC.

Figure 2 shows the effect of these lipid-containing dry eye formulations on cell protection. With a near $0 \% \mathrm{HCEC}$ metabolic activity at the $18 \mathrm{~h}$ recovery interval, solution \#1 and solution \#2 did not protect cells from desiccation stress. Solution \#3, however, offered some protection against desiccation stress.

\section{Effect of Dry Eye Products on Cell Viabilty}

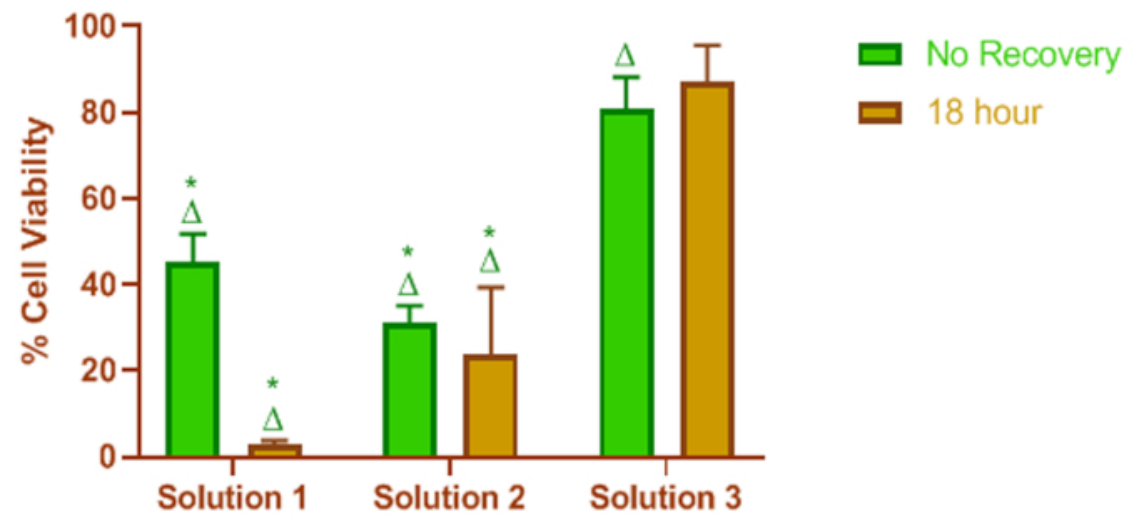

Figure 1: Effect of dry eye lipid enhanced products on cell viability. Relative viability measured by HCEC metabolic activity after treatment with lipid-containing dry eye products. ${ }^{*}=$ statistical significance $(p<0.05)$ relative to solution $\# 3, \Delta=$ statistical significance $(p<0.05)$ relative to the media control for the appropriate recovery period. The height of the bar represents the mean value, and the error bars are the SD. Please click here to view a larger version of this figure. 


\section{Desiccation Protection}

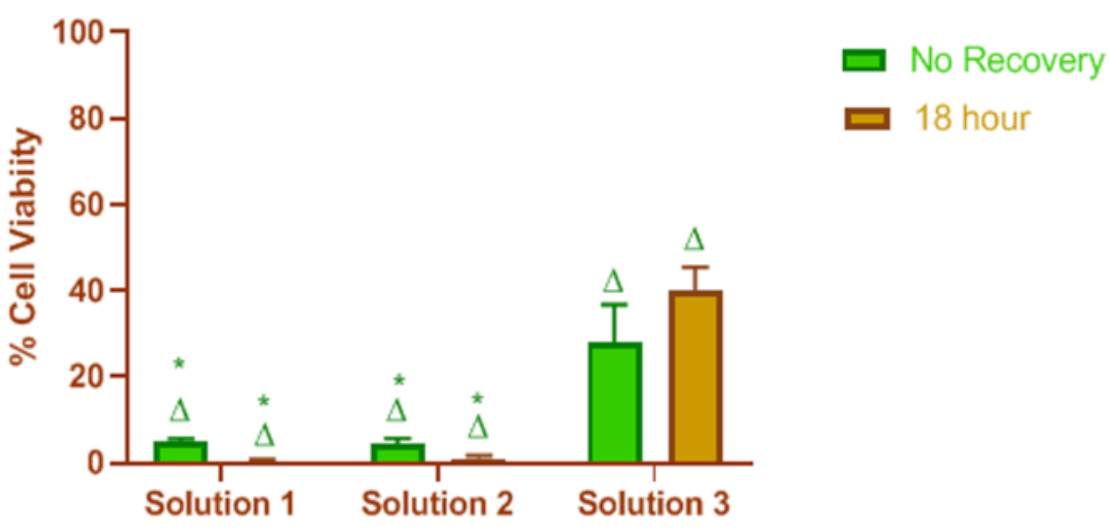

Figure 2: Effect of dry eye lipid enhanced products on desiccation protection. Effect of lipid-containing dry eye formulations on cell protection. Solution \#1 and solution \#2 did not protect cells from desiccation stress, and they did not recover from desiccation effects over time. In contrast, solution \#3 offered some protection against desiccation stress. ${ }^{*}=p<0.05$ relative to solution $\# 3$, and $\Delta=p<0.05$ relative to untreated (control) media for the appropriate recovery time. The height of the bar represents the mean value, and the error bars are the SD. Please click here to view a larger version of this figure.

\section{Discussion}

This protocol is designed to determine the protective effects of artificial tear solutions against cell desiccation. Initially, the cells are exposed to the dry eye drops to coat the cells. Next, the cells are dried, and the metabolic activity is monitored to assess whether the formulations can mitigate the detrimental effects of desiccation stress. These steps are critical for understanding the overall impact the dry eye formulations could have on the overall viability of corneal epithelial cells.

After most of the dry eye formulations are removed from the culture wells, the chemistry of the remaining molecules that are in contact with the cells will impact cell desiccation. Some products contain micro-emulsions of oils that minimize cell evaporation ${ }^{20}$. A study that evaluated friction analysis after rinsing showed maintenance of low friction for some dry eye formulations which may be an indicator of the enhanced residence time of the dry eye formulations ${ }^{21}$. One of the limitations of this assay is that although desiccation protection can be evaluated in comparison to other eye drop formulations the duration of this protection cannot be determined due to the short drying time assessed. Longer drying times in this in vitro assay may require the use of multilayer cultures that have overall more moisture content than a cell monolayer.

The specific incubation times for the formulations on the cells and the duration of drying time may need to be adjusted if temperature and humidity values are changed. Selecting a temperature, $\mathrm{RH}$ and air flow for simulating environmental stress conditions that can contribute to dry eye can be difficult as the seasonal and local environmental conditions are quite variable ${ }^{21}$. Modern-day environment chambers for human studies can vary the temperature between $5{ }^{\circ} \mathrm{C}$ and $35{ }^{\circ} \mathrm{C}$, and $\mathrm{RH}$ between $5 \%$ and $95 \%{ }^{22}$. Using an evaporimeter and tightly fitted goggles it was determined that at $\mathrm{RH}$ of $40-45 \%$ the evaporation rate from the cornea was $0.037 \mu \mathrm{L} / \mathrm{cm}^{2} / \mathrm{min}$ and at $\mathrm{RH}$ of $20-25 \%$ the evaporation rate was $0.065 \mu \mathrm{L} / \mathrm{cm}^{2} / \mathrm{min}^{23}$. If the in vitro model is looking at the protective effect of formulations under extremely low RH conditions such as in airplanes ${ }^{15}$, deserts, or dry indoor environments ${ }^{16}$, incubation times may need to be reduced to accommodate the enhanced evaporation rates from the cells. In the human tear fluid, a variety of lipids derived from meibomian and other glands are present that protect the tears from evaporation. These lipids have different melting points ${ }^{24}$. Temperature changes could impact the fluidity of the tear fluid so tests performed at ambient temperature could have significantly different evaporation rates than tests conducted at $37^{\circ} \mathrm{C}$. Since the average ocular surface temperature range is from 32.9 to $36^{\circ} \mathrm{C}^{25}$, performing the test near this temperature range is recommended.

In addition to the metabolic dye (alamarBlue) used in this protocol, there are other chemical reagents that can be used to assess the effects of product formulations on cell metabolic activity. The tetrazolium salts (MTT, XTT, MTS, WST) are alternative chemicals that can be used. The difference in the tetrazolium salts is that MTT is a positively charged molecule so it can enter the cytoplasm of living cells ${ }^{26}$. MTT becomes insoluble after its reduction by $\mathrm{NAD}(\mathrm{P}) \mathrm{H}^{27}$. It must be solubilized prior to reading absorbance on a plate reader. The other tetrazolium salts are negatively charged ${ }^{28}$. They must be reduced by secondary molecules outside the living cell because they cannot enter the cell and interact directly with intracellular molecules ${ }^{29}$. For the XTT, MTS, and WST-1 assays, the addition of an electron coupling agent 1-methoxy phenazine methosulfate (PMS) can improve the performance of the assays ${ }^{30}$. In contrast to the metabolic assays that use tetrazolium salts, alamarBlue does not require an additional solubilizing or electron coupling agents. Also, unlike XTT, MTS or WST-1, alamarBlue penetrates cell membranes and can be reduced directly by cellular enzymes ${ }^{27,29}$. Direct comparison of alamarBlue to tetrazolium salts have shown that alamarBlue is more sensitive for evaluating the metabolic activity of various cell lines including HCEC $\mathrm{H}^{3,27,31,32}$

Other biochemical endpoints can also be considered for evaluating the protection of human corneal epithelial cells by desiccation. Fluorescent dyes can be used to detect cell membrane permeability and cell esterase activity ${ }^{1}$. Also, apoptosis can be detected by staining for apoptotic markers on the cell surface or by measuring for caspase activity ${ }^{1,33}$. Other assays have determined the effects of ophthalmic products on HCEC tight junctions ${ }^{34}$. These assays could be incorporated in future assessments utilizing the desiccation procedures described in this article.

There are many causes of dry eye. Dry eye can be caused by decreased secretion of tears, increased ocular surface inflammation or increased dehydration at the ocular surface. For the individuals that have evaporative dry eye use of a dry eye drop that prevents their cells from drying 
during desiccation conditions may alleviate the symptoms of dry eye. One of the problems in some dry eye formulations is the presence of preservatives that may harm the ocular surface. Injuring cells is not helpful to dry eye patients as it can cause more cells to die as injured cells are more susceptible to desiccation stress ${ }^{35}$.

A recent review article by Garrigue et al. ${ }^{36}$ provides an assessment of the current knowledge of how lipid-based products act to alleviate evaporative dry eye. Adding lipids to lipid deficient tears can prevent evaporation, thus protecting the cells from desiccation. In addition, humectants are molecules that attract and retain water at the ocular surface ${ }^{37}$. Both glycerin in solution \#1 and propylene glycol in solution \#3 are humectants. Solution \#3 showed a greater protective effect of the HCEC that may have been due to the lipid and humectant properties of the formulation ingredients.

This protocol is designed to evaluate the metabolic activity of HCEC after exposure to the dry eye formulations and to desiccation stress. By using the methods described in this article, new nonirritating and protective eye drops can be developed for people that suffer from evaporative dry eye.

\section{Disclosures}

Three authors (Rekha Rangarajan, Howard A. Ketelson, Lakshman Subbaraman) are employees of Alcon. Alcon is the sole source of funding and sponsor of this project. Lyndon W. Jones, over the past 3 years, has received research support or lectureship honoraria from the following companies: Alcon, Allergan, Contamac, CooperVision, GL Chemtec, Inflamax Research, J\&J Vision, Menicon, Nature's Way, Novartis, PS Therapy, Santen, Shire, SightGlass and Visioneering. Lyndon Jones is also a consultant and/or serves on an advisory board for Alcon, CooperVision, J\&J Vision, Novartis and Ophtecs. The following list of authors have nothing to disclose: Richard Do, David J. McCanna, Adeline Suko, Daryl Enstone, Jaya Dantam.

\section{Acknowledgments}

The authors thank Alcon for providing financial support for these studies.

\section{References}

1. Xu, M., Sivak, J. G., McCanna, D. J. Comparison of the effects of ophthalmic solutions on human corneal epithelial cells using fluorescent dyes. Journal of Ocular Pharmacology and Therapeutics. 29 (9), 794-802 (2013).

2. Maurer, J. K. et al. Quantitative measurement of acute corneal injury in rabbits with surfactants of different type and irritancy. Toxicology and Applied Pharmacology. 158 (1), 61-70 (1999).

3. Xu, M. L., McCanna, D. J., Sivak, J. G. Use of the viability reagent PrestoBlue in comparison with alamarBlue and MTT to assess the viability of human corneal epithelial cells. Journal of Pharmacological and Toxicological Methods. 71, 1-7 (2015).

4. Slaughter, M. R., Bugelski, P. J., O'Brien, P. J. Evaluation of alamar blue reduction for the in vitro assay of hepatocyte toxicity. Toxicology in Vitro. 13 (4-5), 567-569 (1999).

5. Hakkarainen, J. J. et al. Acute cytotoxic effects of marketed ophthalmic formulations on human corneal epithelial cells. International Journal of Pharmaceutics. 511 (1), 73-78 (2016).

6. Paulsen, K., Maile, S., Giebel, J., Tost, F. Lubricating agents differ in their protection of cultured human epithelial cells against desiccation. Medical Science Monitor. 14 (6), PI12-PI16 (2008).

7. Tost, F., Keiss, R., Grossjohann, R., Jurgens, C., Giebel, J. Effect of different artificial tears against desiccation in cultured human epithelia cells. Medical Science Monitor. 18 (5), BR188-192 (2012).

8. Ubels, J. L. et al. Pre-clinical investigation of the efficacy of an artificial tear solution containing hydroxypropyl-guar as a gelling agent. Current Eye Research. 28 (6), 437-444 (2004).

9. Hovakimyan, M. et al. Evaluation of protective effects of trehalose on desiccation of epithelial cells in three dimensional reconstructed human corneal epithelium. Current Eye Research. 37 (11), 982-989 (2012).

10. Zheng, X., Goto, T., Shiraishi, A., Ohashi, Y. In vitro efficacy of ocular surface lubricants against dehydration. Cornea. 32 (9), $1260-1264$ (2013).

11. Matsuo, T. Trehalose protects corneal epithelial cells from death by drying. British Journal of Ophthalmology. 85 (5), $610-612$ (2001).

12. Zheng, Q. et al. Reactive oxygen species activated NLRP3 inflammasomes initiate inflammation in hyperosmolarity stressed human corneal epithelial cells and environment-induced dry eye patients. Experimental Eye Research. 134, 133-140 (2015).

13. Hua, X. et al. Protective Effects of L-Carnitine Against Oxidative Injury by Hyperosmolarity in Human Corneal Epithelial Cells. Investigative Ophthalmology \& Visual Science. 56 (9), 5503-5511 (2015).

14. Schulze, U. et al. Trefoil factor family peptide 3 (TFF3) is upregulated under experimental conditions similar to dry eye disease and supports corneal wound healing effects in vitro. Investigative Ophthalmology \& Visual Science. 55 (5), 3037-3042 (2014).

15. Giaconia, C., Orioli, A., Di Gangi, A. Air quality and relative humidity in commercial aircrafts: An experimental investigation on short-haul domestic flights. Building and Environment. 67, 69-81 (2013).

16. van Setten, G., Labetoulle, M., Baudouin, C., Rolando, M. Evidence of seasonality and effects of psychrometry in dry eye disease. Acta Ophthalmologica. 94 (5), 499-506 (2016).

17. Valtink, M., Donath, P., Engelmann, K., Knels, L. Effect of different culture media and deswelling agents on survival of human corneal endothelial and epithelial cells in vitro. Graefe's Archive for Clinical and Experimental Ophthalmology. 254 (2), 285-295 (2016).

18. Zachari, M. A. et al. Evaluation of the Alamarblue Assay for Adherent Cell Irradiation Experiments. Dose-Response. 12 (2), $246-258$ (2014).

19. Griffith, M. et al. Functional human corneal equivalents constructed from cell lines. Science. 286 (5447), 2169-2172 (1999).

20. Benelli, U. Systane lubricant eye drops in the management of ocular dryness. Clinical Ophthalmology. 5, 783-790 (2011).

21. Davis, R. E., McGregor, G. R., Enfield, K. B. Humidity: A review and primer on atmospheric moisture and human health. Environmental Research. 144 (Pt A), 106-116 (2016) 
22. Calonge, M. et al. Controlled Adverse Environment Chambers in Dry Eye Research. Current Eye Research. 43 (4), 445-450 (2018).

23. McCulley, J. P., Uchiyama, E., Aronowicz, J. D., Butovich, I. A. Impact of evaporation on aqueous tear loss. Transactions of the American Ophthalmological Society. 104, 121-128 (2006).

24. Bron, A. J., Tiffany, J. M., Gouveia, S. M., Yokoi, N., Voon, L. W. Functional aspects of the tear film lipid layer. Experimental Eye Research. 78 (3), 347-360 (2004).

25. Purslow, C., Wolffsohn, J. S. Ocular surface temperature: a review. Eye \& Contact Lens. 31 (3), 117-123 (2005).

26. Stepanenko, A. A., Dmitrenko, V. V. Pitfalls of the MTT assay: Direct and off-target effects of inhibitors can result in over/underestimation of cell viability. Gene. $574(2), 193-203(2015)$.

27. Stockert, J. C., Horobin, R. W., Colombo, L. L., Blazquez-Castro, A. Tetrazolium salts and formazan products in Cell Biology: Viability assessment, fluorescence imaging, and labeling perspectives. Acta Histochemica. 120 (3), 159-167 (2018).

28. Berridge, M. V., Herst, P. M., Tan, A. S. Tetrazolium dyes as tools in cell biology: new insights into their cellular reduction. Biotechnology Annual Review. 11, 127-152 (2005).

29. Rampersad, S. N. Multiple Applications of Alamar Blue as an Indicator of Metabolic Function and Cellular Health in Cell Viability Bioassays. Sensors. 12 (9), 12347-12360 (2012).

30. Li, J., Zhang, D. L., Ward, K. M., Prendergast, G. C., Ayene, I. S. Hydroxyethyl disulfide as an efficient metabolic assay for cell viability in vitro. Toxicology in Vitro. 26 (4), 603-612 (2012).

31. Koyanagi, M., Kawakabe, S., Arimura, Y. A comparative study of colorimetric cell proliferation assays in immune cells. Cytotechnology. 68 (4), 1489-1498 (2016)

32. Uzunoglu, S. et al. Comparison of XTT and Alamar blue assays in the assessment of the viability of various human cancer cell lines by AT-101 (-/- gossypol). Toxicology Mechanisms and Methods. 20 (8), 482-486 (2010).

33. Mcllwain, D. R., Berger, T., Mak, T. W. Caspase functions in cell death and disease. Cold Spring Harbor Perspectives in Biology. 7 (4), a026716 (2015).

34. McCanna, D. J., Harrington, K. L., Driot, J. Y., Ward, K. W., Tchao, R. Use of a human corneal epithelial cell line for screening the safety of contact lens care solutions in vitro. Eye \& Contact Lens. 34 (1), 6-12 (2008).

35. Puhlev, I., Guo, N., Brown, D. R., Levine, F. Desiccation tolerance in human cells. Cryobiology. 42 (3), $207-217$ (2001).

36. Garrigue, J. S., Amrane, M., Faure, M. O., Holopainen, J. M., Tong, L. Relevance of Lipid-Based Products in the Management of Dry Eye Disease. Journal of Ocular Pharmacology and Therapeutics. 33 (9), 647-661 (2017).

37. Lievens, C. et al. Evaluation of an enhanced viscosity artificial tear for moderate to severe dry eye disease: A multicenter, double-masked, randomized 30-day study. Contact Lens \& Anterior Eye. 42 (4), 443-449 (2019). 